\title{
ADHD Influences throughout a Diagnosed Person's Lifespan A Literature Review
}

\author{
Dr. Phillip D. Clingan
}

\begin{abstract}
The qualitative review discusses Attention Deficit Hyperactivity Disorder (ADHD) and the developmental influences of ADHD throughout a diagnosed person's lifespan. The literature review examines and contrasts the negative and specific impacts of ADHD. A small literature review is focusing on the issue acted on elementary and secondary sources, which retrieved from education archives and diverse psychological sources of data such as University libraries. The central question behind the research is what challenges does a person diagnosed with ADHD face as they begin the various stages of lifespan? According to the literature review, ADHD is a compound and heterogeneous neurodevelopment disorder with no curative treatment. The disorder encompasses cognitive-behavioral components, which include inappropriate stages of inattention, hyperactivity, and impulsivity. The review provides an academic platform for scholars and clinical psychologists. Despite limited studies of aging diagnosed patients, considerable advances toward understanding the disorder provide coping strategies throughout all lifespans of human development. Most ADHD practices are in a continual state of adjustment and progress, as evidenced by developments as evidence in the limited studies considering the specifics regarding patients between ages $50-70$ and morbidity challenges. There is a substantial demand for future research on the overall impacts and contrasts each person diagnosed with ADHD face throughout the lifespan. Each diagnosed person sufferers' different spectrums of the disorder throughout the development of life. Further research will yield the challenging light on the different stages of life, and to further improve therapies for obesity, sleep apnea, depression, diminished employment, untimely deaths from accidents, and diminished quality of life.
\end{abstract}

Index Terms - Attention Deficit Hyperactivity Disorder, Substance Use Disorders, Lifespan Psychology, Early Years, Childhood Lost, Storm and Stress, World is Your Oyster, Mid-life Crisis, Golden Years, and Need for Future Research.

\section{INTRODUCTION}

Highlight a Developmental psychology, likewise recognized as Lifespan Development, is the scientific examination of human development and refers to how people evolve, and remain the same, from conception to death. A comprehensive approach within the field of psychology to explain the cognitive, physiological, emotional, and social developments through a person's lifespan [6]. The capacity to identify a human being's development throughout the lifespan is central to psychology due to it being capable to explain what advances a human being's development in the lifespan and what sets back improvements in the lifespan. A considerable

Dr. Phillip D. Clingan, Criminal Justice \& Social Sciences, Pierce College/ Lakewood WA., United States portion of theories within this discipline concentrates on development during childhood, as this stage develops the most change during a human being's lifespan [10]. There are many factors which individuals must progress through which serve to develop and present challenges which diagnosed people must progress through until the conclusion of the lifespan.

Attention deficit hyperactivity disorder is a disability which many people develop in the lifespan. Attention, deficit hyperactivity disorder, is a disability which can have many implements on a human being's stages of life. Conception, infancy, childhood, adolescence, early adulthood, middle age, and indeed older age are all of the stages of lifespan which can be influenced by attention deficit hyperactivity disorder.

When examining a human being's lifespan, what challenges does a person diagnosed with ADHD face as they begin the various stages of lifespan?

\section{1) Purpose Statement}

The intention of the paper is to evaluate the challenges that a person diagnosed with attention deficit hyperactivity disorder experiences throughout the lifespan. The paper will examine sources to examine and assess these challenges and how they influence the individual's development within the lifespan. The topics examined are how attention deficit hyperactivity disorder affects a human being's childhood, the challenges faced throughout young adult and adulthood, and addiction. Meantime, outlining the sources intended to present evidence which will support the analysis concern that attention deficit hyperactivity disorder poses considerable challenges that a diagnosed individual could face throughout the lifespan.

\section{LITERATURE REVIEW}

\section{1) Lifespan Psychology}

Attention-deficit/hyperactivity disorder (ADHD) is a complicated, chronic, and multifactorial neurodevelopment disorder [8]. For some, the disorder affects many challenges throughout an individuals' lifespan. The attention deficit hyperactivity disorder has three primary manifestations, a) hyperactivity, b) inattention, and c) impulsivity [11]. The manifestation of hyperactivity happens when an individual tends to be energetic, restless or is incapable to remain still. The manifestation of inattention is where an individual is incapable to concentrate and lack mental attention for further prolonged periods. The manifestation of impulsivity occurs when an individual tends to physically act before thinking, acting on impulse such as disturbing people in the middle of a 
conversation [6]. Individuals diagnosed with attention deficit hyperactivity disorder can experience many diverse challenges to manage throughout the various stages of the lifespan.

\section{2) The Early Years and Diagnoses}

Kail and Cavanaugh, 2019 assert children are more prone to be diagnosed with ADHD. Specifically, if, as preschoolers, they were extraordinarily overactive or had torrents of temper. During the adolescence lifespan stage inattention and impulsivity remain, but hyperactivity often diminishes. Children with hyperactivity symptoms tend to be highly energetic and restless. Children with impulsivity symptoms tend to act before they think while children exuding mental inattention tend to have trouble focusing. Specifically, and comparatively, males $12 \%$ more than females, $4.7 \%$ are diagnosed with ADHD based on a 2011 National Health Interview Survey [7]. Some children have one or two of the core symptoms, while other children exhibit all three of the core symptoms. Children with these core symptoms can struggle with schoolwork, be confused, are 'regularly on the move,' or indeed have reduced/no danger awareness [16]. Young diagnosed individuals attempt to manage or self-regulate these symptoms throughout the lifespan.

\section{3)Childhood Unconscious}

In the United States, about $6 \%$ of all school-age children are diagnosed with ADHD to comparatively 8\%-12\% globally [7].Hamed, Kauer, and Stevens, 2015 assert the diagnosis is most regularly performed in middle childhood when its manifestations become visible. For example, the child cannot mentally concentrate on schoolwork, presents physical disruptive behaviors in school and home, and struggle to socialize and create friends. The risk factor markers of ADHD are observed in the preschool years [6]. Children with ADHD can be academically delayed, and are negatively labeled or treated negatively, and looked at as problematic instead of being seen as a child with a problem. During this stage in life, children struggle to mentally concentrate, remain physically still, and process thoughts before physically acting, which can cause a school environment challenging [4]. Social interactions and making friends are negatively influenced by these challenges. Children tend to have

- a high degree of physical activity;

- act impulsively;

- are not cognizant of personal space;

- isolate themselves from others;

- low self-esteem;

- $\quad$ self-report feeling different from other children;

- faced with taking medicine;

- experience repeated failed experiences;

- labeled as intellectually challenged,

and are incapable to play or communicate for periods greater than a few minutes, which can generate problems within a child's social interaction [16].

\section{4)Perfect Storm}

Attention, deficit hyperactivity disorder can occur through differing levels of lethargy, inattention, and hyperactivity [5].
Which anyone of these areas of concern could prevent an individual in school from being academically successful. Additionally, it could present challenges to students diagnosed with ADHD, because as young adults, few individuals with ADHD complete college, and many have work-related complications [6]. Another challenge that individuals diagnosed with ADHD could experience in the lifespan is nicotine dependence. In most studies, ADHD is recognized as an independent risk factor regarding Nicotine Dependence. Smokers diagnosed with ADHD self-report higher progression and prevalence regarding percentages of smoking and enhanced difficulty when quitting. Few studies exist concerning ADHD and smoking habits, mainly how nonsmokers discuss motivational factors and how nonsmoker differentiate from diagnosed ADHD smokers. The relationship could be explained by dopaminergic dysregulation, which is observed in addictive disorder, which supports the association both in nicotine dependency and ADHD [9].

\section{5)Anything is Possible}

One of the principal treatments of attention deficit hyperactivity disorder is medical prescription, like stimulants, which can be extremely addictive. The prescribed stimulant increases neurotransmitter levels, blood pressure, and heart rate while decreasing sleep and appetite (Center for Disease Control and Prevention, 2019). Some commonly prescribed stimulants to manage ADHD include

- Adderall (amphetamine)

- Concerta (methylphenidate)

- Daytrana (methylphenidate patch)

- Dexedrine or Dextrostat (dextroamphetamine)

- Focalin (dexmethylphenidate)

- Metadate or Methylin (methylphenidate)

- Ritalin (methylphenidate)

- Vyvanse (lisdexamfetaminedimesylate)

Statistically, $25 \%-55 \%$ of adults diagnosed with ADHD have a past of narcotics abuse/dependencies [1]. The existence of ADHD represents more than two-fold increased susceptibility to developing substance abuse disorder (SUD). Additionally, ADHD diagnose subjects have been reported to be susceptible to earlier onset of substance abuse, and more challenging remains in treatment and rehabilitation [12]. Stimulants mentioned above are applied to encourage the parts of the brain which in turn inhibit impulsive and excitable behavior, which is why stimulants are employed to manage attention deficit hyperactivity disorder in an individual diagnosed with the disorder [6]. More typically, due to stimulants being highly addictive, and in partnership with the adult individuals with the disorder being at a greater risk for substance use disorders (SUD), the potential combination can contribute to an adult with the disorder becoming highly addicted to narcotics, alcohol, stimulants or other addictive stimulants.

\section{6)Mid-Life Juncture}

Individuals diagnosed with ADHD face challenges with employment in the adulthood stage of the lifespan [5].Individuals suffering from symptoms during this stage of life experience challenges such as finishing school and 
college. Such challenges decrease the probability of progress toward finding jobs even when the diagnosed person is qualified to perform. Blue Collar adults suffering from ADHD experience higher ratios of work-related issues, and social sector's [3]. Many jobs demand a high school diploma or a college degree. Due to the many positions that require degrees, the individuals diagnosed with ADHD and unable to complete either degree mentioned would most likely experience high ranges of difficulty getting jobs even if the sufferer is qualified.

\section{7) The Golden Years}

The validation of ADHD among older adults has developed into an increasing concern in the fields of psychology and medicine. Primarily due to a growing quantity of adults aged 50-60 plus years seeking assessment for ADHD for the first time. Presently, there is a limited amount of research on ADHD in adults aged 50-60 years and older [5]. The epidemiology suggests there are $2.5 \%$ of the aging population are underdiagnosed with respect to ADHD. More notably, during the Golden Years age groups, the psychiatric comorbidity levels are abnormally high as $60-70 \%$ of diagnosed ADHD adults suffer from the comorbid disorder [5].

The age group 50-70 diagnosed with ADHD can likewise be identified with medical comorbidity. Particularly, experiencing a heightened risk of sleep apnea, obesity, and asthma [5]. Attention, deficit hyperactivity disorder, can decrease somewhat as an individual becomes older. Some manifestations, like hyperactivity, can begin to diminish as time passes on, but impulsivity and inattention might decline somewhat, ultimately tend to remain the same [6]. Even as an individual with this disorder grows older, the manifestations of the disorder remain. Older adults with ADHD will remain impaired, and even while aging, they may not exhibit all of the benchmarks for the diagnosis of the disorder [6].

Additionally, there are probable consequences for this specific aging group of untreated ADHD. Some studies examine the long-term results of adults, while new studies examine childhood ADHD outcomes. Geffen and Foster discuss and list possible consequences. They include

- impaired relationships;

- impaired quality of life;

- premature death from accidents;

- reduced employment;

- suicide;

- vulnerability to addiction;

- vulnerability to depression and apprehension.

\section{CONCLUSION AND NEEDS FOR ADDITIONAL RESEARCH}

Overall, the analysis paper concluded the literature review objective of identifying what challenges does a person diagnosed with ADHD face as they begin the various stages of lifespan. People diagnosed with ADHD face many alternative challenges throughout their full lifetime. Throughout the early years, childhood, young adult, middle-age adulthood, and golden years, a person will encounter issues with education, social interactions, and even addictions/dependencies and medical comorbidity. These are a number of the challenges that people diagnosed with attention deficit hyperactivity disorder will face throughout their lifetime.

In the long run, this subject requires future analysis to reveal the long-run challenges that may result from an aging person's diagnosing of attention deficit hyperactivity disorder. Additional analysis is necessary as a result of ADHD poses a rather broader vary of issues that not each individual with this disorder faces. Attention deficit hyperactivity disorder is disabling and continued research helps address the prevalent comorbidity while reducing disabilities. Future research could offer improved treatments for obesity, sleep apnea, depression, reduced employment, premature deaths from accidents, and impaired quality of life.

\section{ACKNOWLEDGMENT}

I am using this opportunity to express my gratitude to my family and friends who supported me throughout the course of this research project. I am thankful for their aspiring guidance, invaluably constructive criticism and friendly advice during the project work. I am sincerely grateful to them for sharing their truthful and illuminating views on a number of issues related to the project.

\section{REFERENCES}

[1] Addiction Center, \&Juergens, J. (2016, March 1). ADHD and Addiction. $\quad$ Retrieved from https://www.addictioncenter.com/addiction/adhd/

[2] Center for Disease Control and Prevention. (2019, April 5). ADHD Treatment Recommendations. Retrieved from https://www.cdc.gov/ncbddd/adhd/guidelines.html

[3] Corcoran, J., Schildt, B., Hochbrueckner, R., \& Abell, J. (2017) Parents of children withattention Deficit/Hyperactivity Disorder: A meta-synthesis, part II: C \& A C \& A. Child\& Adolescent Social Work Journal, 34(4), 337-348 doi:http://ezproxy.pierce.ctc.edu:2101/10.1007/s10560-017-0497-1

[4] Hamed, A. M., Kauer, A. J., \& Stevens, H. E. (2015). Why the Diagnosis of Attention DeficitHyperactivity Disorder Matters. Frontiers in Psychiatry, 6, 168 doi: $10.3389 /$ fpsyt. 2015.00168

[5] Josh Geffen, \& Kieran Forster. (2018). Treatment of adult ADHD: a clinical perspective. SAGE publications, 8(1), 25-32. Retrieved from https://www.ncbi.nlm.nih.gov/pmc/articles/PMC5761907/

[6] Kail, R. V., \& Cavanaugh, J. C. (2019). Human Development: A Lifespan View (8th ed.). Boston, MA: Cengage Learning.

[7] Luo, Y., Weibman, D., Halperin, J. M., \& Li, X. (2019). Attention Deficit HyperactivityDisorder (ADHD). Frontier Human Neuroscience. $\quad$ Retrieved from https://doi.org/10.3389/fnhum.2019.00042

[8] Martinez-Badía, J. (2015). Who says this is a modern disorder? The early history of attention deficit hyperactivity disorder. World Journal o Psychiatry, 5(4), 379.doi:10.5498/wjp.v5.i4.379

[9] Mitchell, J. T., McIntyre, E. M., McClernon, F. J., \& Kollins, S. H. (2014). Smoking Motivation in Adults with Attention-Deficit/Hyperactivity Disorder Using the Wiscons in Inventoryof Smoking DependenceMotives. Nicotine \& Tobacco Research, 16(1), 120-125.doi:10.1093/ntr/ntt144

[10] Mcleod, S. (2017). Developmental Psychology. Retrieved June 19, 2019 , https://www.simplypsychology.org/developmental-psychology.html

[11] National Institute of Mental Health. (2016, March). Attention-Deficit/Hyperactivity Disorder. Retrieved fromhttps://www.nimh.nih.gov/health/topics/ attention-deficit-hyperactivity-disorder-adhd/index.shtml

[12] Nogueira, M., Bosch, R., Valero, S., Gómez-Barros, N., Palomar, G., Richarte, V., . . . Ramos-Quiroga, J. (2014). Early-age clinical and developmental features associated withsubstance use disorders in attention- Deficit/Hyperactivity disorder in adults. Comprehensive Psychiatry, 55(3), 639-49.

[13] Verkuijl, N., Perkins, M., \& Fazel, M. (2015). Childhood attention-deficit/hyperactivity disorder. BMJ : British Medical Journal (Online),

350

doi:http://ezproxy.pierce.ctc.edu:2101/10.1136/bmj.h2168 


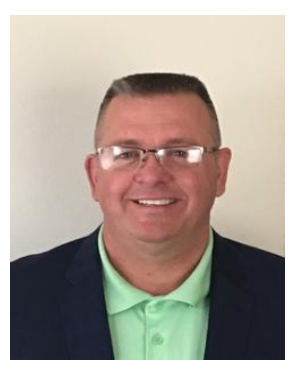

Dr. Clingan after serving 21 years in the United States Army undertook his Psy.D in the Department of Psychology, specializing in criminology in the School of Organizational Leadership at Ashford University, Denver, Colorado. He is a faculty member teaching in the Departments of Psychology and Criminal Justice in San Antonio, Texas for Pierce College and serves as a content expert on dissertation committees for Grand Canyon University School of Doctoral Studies regarding quantitative studies for doctoral candidates. Dr. Clingan specializes in preeminent psychology as applied within the field of criminology. His psychology work is based upon field experience in criminal trials since 2013 and heavily influenced by the theoretical frameworks of Dr. Paul Ekman and measuring nonverbal communication. Loves writing and riding his Indian Motorcycle. 